\title{
"It's like a puppet master": User Perceptions of Personal Autonomy when Interacting with Intelligent Technologies
}

\author{
Supraja Sankaran \\ Department of Industrial Design, \\ Eindhoven University of Technology \\ Netherlands \\ s.sankaran@tue.nl
}

\author{
Panos Markopoulos \\ Department of Industrial Design, \\ Eindhoven University of Technology \\ Netherlands \\ p.markopoulos@tue.nl
}

\begin{abstract}
Applications which use some form of artificial intelligence (AI) have become embedded in our everyday interactions. Very often, AI-based apps are personalized and modelled on users' needs and preferences. However, such applications of AI tread a delicate balance between enhancing user experience and jeopardizing personal autonomy. Personal autonomy and sense of agency are crucial for human well-being and development. In this paper, we probe this fine balance aiming to capture users' lived experiences and perceptions of interacting with AI-based apps. We present insights from a phenomenological study $(\mathrm{N}=15)$ regarding users' perception of personal autonomy when interacting with $\mathrm{AI}$ in everyday contexts. We found that these experiences are transitory and largely influenced by contextual factors. Users experience a loss of autonomy when their privacy or identity is threatened or when their expectations are broken. To mitigate such loss of autonomy, mechanisms for providing intelligibility and control of AI are desired.
\end{abstract}

\section{CCS CONCEPTS}

- Computing methodologies $\rightarrow$ Philosophical/theoretical foundations of artificial intelligence; $\bullet$ Human-centered computing $\rightarrow$ User studies.

\section{KEYWORDS}

human-AI interaction, autonomy, phenomenology, user experience

\section{ACM Reference Format:}

Supraja Sankaran and Panos Markopoulos. 2021. "It's like a puppet master": User Perceptions of Personal Autonomy when Interacting with Intelligent Technologies. In Proceedings of the 29th ACM Conference on User Modeling, Adaptation and Personalization (UMAP '21), fune 21-25, 2021, Utrecht, Netherlands. ACM, New York, NY, USA, 11 pages. https://doi.org/10.1145/ 3450613.3456820

\section{INTRODUCTION}

Writers and creators of science fiction have long been using representations of artificially intelligent and sentient agents as an instrument to present both utopian and dystopian perspectives.

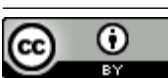

This work is licensed under a Creative Commons Attribution International 4.0 License.

UMAP '21, June 21-25, 2021, Utrecht, Netherlands

(C) 2021 Copyright held by the owner/author(s)

ACM ISBN 978-1-4503-8366-0/21/06.

https://doi.org/10.1145/3450613.3456820
A snippet from a famous scene in the movie Matrix [58] goes as follows-

Neo: You already know which choice I'm going to make!

Oracle: Wouldn't be an Oracle if I didn't.

Neo: So the point?

Oracle: You are not here to know the choice. You are

here to know "why" you made it.

We can easily draw an analogy from the exchange above to the interaction between humans and AI, with the user in the role of Neo and the Oracle as an AI system which predicts the user's choices. The need to know "why" is a key ambition for research into explainable AI. Perhaps less widely recognized, but equally important is the need for autonomy. Further in the movie the Oracle says, "choice is an illusion shown by people in power". Could this be how users feel when interacting with AI?

Giving users sufficient choice and control in order to enable their personal autonomy and agency have been long discussed across disciplines is a fundamental human value that is critical for human well-being and development. From a social psychology and human behavior perspective, autonomy refers to empowering humans with a freedom of choice and the ability to determine one's own goals and actions $[14,48,57]$. In the context of human-machine and multi-agent interactions, autonomy can pertain to either the agent or the human having control, and being able to make decisions independently $[1,4,33]$. From a political and societal standpoint, human autonomy is identified as a key value in democratic constitutions [60].

To enhance their capabilities, AI systems are increasingly endowed with greater autonomy to act independently, make decisions, and take-over control from humans. As a consequence, humans interacting with these data-driven autonomous systems may experience a reduced sense of autonomy as they relinquish control and decision-making to them [7]. Human agency and individuals experiencing a loss of control over their lives have been highlighted as a key concern for $\mathrm{AI}$ in the near future by experts [25]. Organizations such as the European Commission have published ethical guidelines for developing trustworthy $\mathrm{AI}$ in which respecting human autonomy has been emphasized as one of the primary principles [11, 42].

Despite the growing interest in human aspects of AI, research regarding how humans experience the loss of personal autonomy due to AI has been fragmented. Autonomy is a complex concept and current discussions focus primarily on specific facets of autonomy such as trust, privacy, control or decision-making support [1, 28, 41, 53] and in quite specific contexts such as automated driving [59] 
or human-robot interactions [45, 47]. For example, Torkamann et al. [53] studied user perceptions of creepiness when interacting with automated recommender systems in three very specific contexts (hotel, movie and health) with the focus primarily only on trust. Sankaran et al. [49] highlighted the concern of autonomy in terms of humans losing control over their goals, decisions and actions when interacting with conversational agents. Therefore, there is a need to take a more comprehensive look into factors that impact the personal autonomy of users when interacting with AI in everyday contexts.

To this end, we report on a study to understand user perspectives of personal autonomy when interacting with common everyday applications that use some form of AI. Since the users' understanding and perceptions of AI are varied, it was important to contextualize AI for participants within their individual 'lived' interaction experiences. Therefore, we used a phenomenology approach [12] with 15 demographically diverse participants, to gather their perspectives when interacting with applications across different contexts. In this paper, we detail the methods and insights gathered from the study and present some directions for future research. We aim for the insights presented in this paper to open up a critical discussion on human autonomy considerations when designing AI systems and pave the way for further research in this direction.

\section{UNDERSTANDING LIVED EXPERIENCES}

The field of human-computer interaction (HCI) has for some time emphasized the importance of understanding and designing the user experience with different technologies [20]. Such efforts are challenged by the level of subjectivity and variation in people's experiences, which are tied to different contexts and make it difficult to synthesize reliably meaningful insights from them. In trying to guide researchers and designers in this field, it is useful to study the essence of this user experience, at least the aspects of it that are shared across individuals and which refer to the feeling of autonomy or loss thereof in relation to the use of AI. Phenomenology as an inquiry method is uniquely positioned to address the disparate nature of people's experiences and to enable researchers to learn from the experience of others [40]. Phenomenology is commonly used in domains such as nursing, pedagogy or psychology, to study the nature of various lived experiences. In the present study, we adopted phenomenology in order to gain a nuanced understanding of peoples' experiences and perceptions of autonomy and agency when interacting with everyday applications that use AI. Specifically, we adopted the transcendental phenomenology approach $[12,39]$, to empirically gain an understanding of this phenomenon, while limiting the influence of the researchers' perspectives and assumptions. There are various approaches and philosophies around phenomenology that range from the traditional interpretive or hermeneutic phenomenology [55] to modern philosophies derived from Ihde's work on post-phenomenology and experimental phenomenology [22, 23]. Nonetheless, for our study, we chose to adopt the transcendental phenomenology approach as proposed by Moustakas [39] for two key reasons-

(1) This method includes explicit steps for bracketing out researchers' individual perspectives and focuses only on the lived experience of participants as originally suggested by
Husserl [21]. It thus allows discovering perspectives of the phenomenon as experienced by the participants without being influenced by existing scientific know-how and assumptions. It also enables researchers to glean new insights and gain an unbiased understanding of the essence of the phenomenon.

(2) The approach also builds on psychological theories and data analysis methods $[10,18,26]$. Specifically, it offers a grounded and structured means to cluster disparate subjective perspectives and infer the essence of perceptions of personal autonomy and human agency when interacting with AI applications.

\subsection{Participant selection}

To gather a rich collection of experiences across a diverse demography of participants, we interviewed 15 participants using a purposive sampling approach combining criterion and maximum variation sampling [35]. While there is no fixed rule regarding the ideal number of participants needed for a phenomenological study, anywhere between 3-25 participants has been recommended [15, 46]. The key criterion for selecting participants was that they would have sufficient experience in using everyday applications that rely on AI to generate options and recommendations. We aimed for a maximum possible variation in demographics, in terms of age, gender, education and profession, particularly with respect to variations in technical knowledge and experience when it comes to AI [Table 1]. Amongst the 15 participants, there were 8 male and 7 female participants (age groups- 25-34:n=13; 35-44:n=1; 45-54: $n=1$ ). All participants had at least a bachelor's degree. Their educational and professional backgrounds included mechanical and automotive engineering, physics, chemistry, biotechnology, computer science, business management, banking and finance.

Table 1: Demography of participants from the phenomenology study.

\begin{tabular}{llll}
\hline ID & Age & Gender & Field of work/education \\
\hline P1 & $25-34$ & M & Material science \\
P2 & $44-55$ & F & Physics, Banking \& Finance \\
P3 & $25-34$ & M & Embedded systems \\
P4 & $25-34$ & M & Mechanical engineering \\
P5 & $25-34$ & F & Computer science \\
P6 & $25-34$ & M & Engineering (Automotive) \\
P7 & $25-34$ & F & Business management \\
P8 & $25-34$ & M & Physics \& Biotechnology \\
P9 & $25-34$ & F & Material engineering \\
P10 & $25-34$ & M & Management \\
P11 & $25-34$ & F & Chemistry \\
P12 & $25-34$ & M & Engineering \\
P13 & $25-34$ & M & Materials science \\
P14 & $25-34$ & F & Finance \\
P15 & $35-44$ & F & Human-computer Interaction \\
\hline
\end{tabular}

\subsection{Phenomenological interview}

We curated a list of 10 everyday application categories that are commonly used and use some form of AI [Table 2]. 
Table 2: Selected list of everyday application categories which use AI

\begin{tabular}{lll}
\hline & Application Category & Examples \\
\hline C1 & Booking \& reservation systems & Booking.com, Kayak, Airbnb etc. \\
C2 & e-Commerce sites/apps & Amazon.com, Bol.com, Flipkart.com etc. \\
C3 & Social media platforms & Facebook, Instagram, Twitter, LinkedIn etc. \\
C4 & Domotics & Nest thermostat, Google home and home hubs etc. \\
C5 & Entertainment apps & Netflix, Spotify, Youtube, Amazon Prime etc. \\
C6 & Navigation apps & Google maps, Uber, Lyft etc. \\
C7 & Auto replies \& profile generation & Gmail auto-compose, messengers, LinkedIn etc. \\
C8 & Health \& Fitness apps & Runkeeper, Strava, Fitbit etc. \\
C9 & News \& weather sites/apps & Google news, Apple news+, Accuweather etc. \\
C10 & Voice agents & Alexa, Siri, Google assistant etc. \\
\hline
\end{tabular}

After approval from the university's ethical review board, we contacted participants who met the sampling criteria and sent them an information and consent form prior to the study. Because of practical considerations relating to the COVID-19 pandemic, interviews were conducted virtually using Google Meet ${ }^{1}$. The interview audio was recorded and the first author used an auto-transcription plugin ${ }^{2}$ to produce an initial transcription of the interviews, which they proofed afterwards. At the start of the interview participants were asked to fill out a brief questionnaire with their demographic information and to rate how frequently they use the above types of applications. They were asked to pick one or two applications with which they interact most frequently and which they use at least everyday in order to ensure that they have sufficient experience to share meaningful insights. The interviews were semi-structured and were aimed at gaining insights in two key aspects as recommended by Moustakas and Polkinghorne [39, 46]- (RQ1) How has their experience been so far when interacting with their chosen application, and (RQ2) How have different contexts or situations influenced their experience. Furthermore, they were also asked if they were aware of the presence of an AI algorithm driving these apps and, if so, what was their understanding of how these algorithms work (RQ3). This was asked primarily to understand if their perception of personal autonomy was influenced by their awareness of AI powering the system. Furthermore, to bracket out researchers' individual perspectives and to avoid introducing a biased conception of autonomy, the term 'autonomy' was not mentioned while interviewing the participants. They were merely asked to share their experiences both positive or negative when interacting with these applications. All interviews were carried out by the same researcher to maintain consistency.

\subsection{Data analysis}

To analyze the interview transcripts and infer the essence of the experiences of participants, we followed the analysis approach as described by Moustakas and Polkinghorne [39, 46]. We started by going through the interview transcripts of each participant to identify significant statements, a process which is referred to as

\footnotetext{
$\overline{{ }^{1} \text { https://meet.google.com/ }}$

${ }^{2}$ https://tactiq.io/
}

"horizonalization" by Moustakas [39]. In this context of understanding user perceptions of interacting with AI applications, we identified significant statements falling in two categories- (a) statements which were specific to an application and (b) statements which were about AI or AI behavior in general [Figure 1]. The statements were color coded to distinguish between positive, negative or mixed experiences. As a next step, all significant statements were grouped in meaningful clusters or "meaning units" [39] from the lens of personal autonomy.

In order to achieve methodological rigour, we adopted a verification and validation approach [34]. Verification was fulfilled by adhering to grounded phenomenological methods, bracketing out researchers' perspectives, selecting a diverse and adequate sample, and interviewing until a saturation was achieved [17]. A within project validation was performed following a grounded data analysis approach [39] of horizonalization and clustering by the primary researcher and using member checks by 3 randomly selected participants to verify the clusters and associated significant statements [10]. Finally, an inter-rater reliability between 2 raters showed a $90 \%$ agreement rate with a $\kappa=0.89 ; 95 \% C I(0.74,1.00)$ [16]. Minor or partial disagreements were mainly because some significant statements were perceived to fit to multiple meaning units. These conflicting mappings were discussed to decide together which mapping lent insights into perceived autonomy and were clustered accordingly.

\section{OUTCOMES}

The phenomenological interviews with participants lasted between 18 - 52 minutes with an average duration per interview of 25 minutes. The total duration of all interviews was 376 minutes. During the horizonalization process, a total of 218 significant statements were identified (positive perspective: 42 , negative perspective: 96 , mixed perspective: 82 ). On average this amounted to 15 significant statements per participant. A certain significant statement or quote was construed positive if it included phrases like- "it's a good thing", "what I really like..", "does right", " I like it" etc. On the contrary, statements which included words or phrases such as "frustrating", "annoying", "it doesn't do..[user expectation]", "I don't like..", "really dangerous", "scary", "it's disturbing", "gives me anxiety" etc. were associated with a negative user perception. A mixed perception was mainly associated to a generic opinion, an explicit middle stance or 


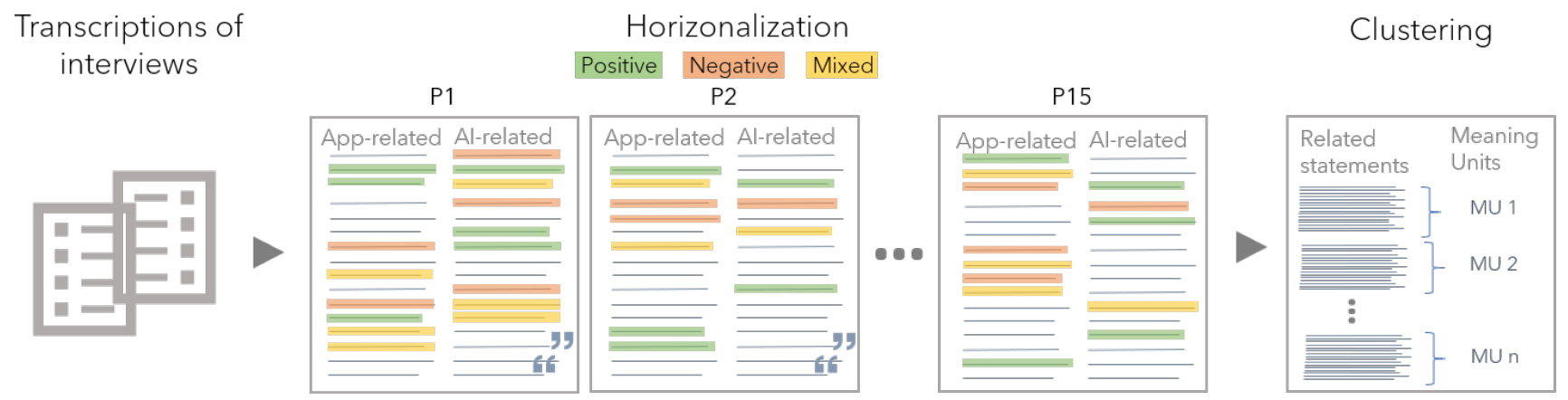

Figure 1: Process of analyzing the interview transcripts.

a future perspective such as "it is both good and bad", "it's kind of like a frenemy", "I think it might..", "it could be.." etc.

Of the 218 significant statements, 95 were specific to the application context and 123 were related to $\mathrm{AI}$ in general. We used both these types of data to address RQ1, application context specific data to address RQ2 and AI related data to address RQ3. From the significant statements 10 meaning units emerged with respect to the impact on autonomy- (MU1) Time-saving, (MU2) Decision-making support, (MU3) Personalization and ease of use, (MU4) Knowledge and transparency, (MU5) Shifting control, (MU6) Freedom of choice, (MU7) Interconnected systems and data sharing, (MU8) Trust and privacy, (MU9) Manipulation or polarization, (MU10) Breaking expectations. We discuss each of these in more detail below.

\subsection{Time-saving}

In different application contexts such as social media, entertainment and news feeds, participants felt that the automation in the applications primarily helped them save time. This was cited as the primary reason for using certain applications or enabling automation features and recommendations within applications. For example, P11 stated that-

"... you feel that they [social media, news feed] surface what you want usually and because you're running out of time and you don't have time to go through all the new posts."

"The good thing is whenever they share a video I can quickly check it out and don't miss videos from them."

[P9 in the context of entertainment apps]

\subsection{Decision-making support}

Complimentary to the time saving aspect, participants also felt that in cases where they have limited prior knowledge or expectation, the automated suggestions or recommendations offered by applications facilitated their own decision making. For example, which movie to watch (C5), what exercise to do (C8) and in unfamiliar contexts such as where to eat in a new city (C6).

"I do like the recommendations that I get in terms of shops or restaurants, especially in a new place where I have no idea what to do." [P6 in the context of navigation apps]

\begin{abstract}
"..to some extent what Netflix does right, because it basically analyzes my viewing habits and throws up similar content. That makes it easy for me to decide what I should be watching next." [P3]
\end{abstract}

However, some users felt the need to have more freedom to a make independent decisions and did not like the suggestions or recommendations made by the applications, neither in the same nor in different contexts.

"I don't want any suggestions. I've never asked for any suggestions. Then why are you voluntarily giving me some suggestions? That's how I feel! [shrugs]" [P2 in the entertainment context]

\subsection{Personalization and ease of use}

In different applications, $\mathrm{AI}$ is mainly used to enhance personalization and tailor the content presented to the users based on their profiles. In most contexts, users appreciated the personalization and expressed a positive sentiment towards it. "..it is suggesting things to me according to my liking..", "it's very easy for me to follow my interests..". This was especially apparent in the entertainment context and domotics where it become easier to delegate redundant tasks of searching for interests or setting preferences.

"It would automatically set the temperature to $23^{\circ}$ based on my preferences [talking about a smart thermostat], and it could predict how long it would take to reach that temperature. Also what I like is, it would always turn off at a certain hour in the night because I opted that before." [P5 in the domotics context]

"It's very easy for me to follow my interests because I don't have to keep searching them again." [P4 in the entertainment context]

However, a couple of participants highlighted that sometimes personalization narrows down or streamlines their options too much. This was perceived in the entertainment context-

"The bad thing is, if I start watching them, you know you restrict yourself to a certain type of content and you rarely venture outside of what you are normally comfortable with.” [P9] 


\subsection{Knowledge and transparency}

Across different contexts, participants felt that applications were not fully intelligible about the information that was presented and why it was being presented to them. While all of them were aware of some form of automation, they still alluded to the black-box nature of these applications and a lack of thorough understanding of how they work. At least 4 participants who were more tech-savvy, commented that they were fairly aware that most information presented was based on their search history or past online behavior and other profiling mechanisms that applications adopted. However, even they felt that it was not always clear which of these specific aspects generated the content they were viewing and expected to have more transparency on that front. The lack of knowledge and transparency on how the system arrived at recommendations were apparent from users using phrases such as "it is not clear", "I'm not sure how it works", "there is something in the background" etc.

"I have something that says suggested by Siri. I don't know what that means. I don't know if that comes from what I asked Siri, but I see 'nutrition', 'health', 'fashion accessories'. I don't think I ever asked anything about fashion accessories to Siri. So it's very unclear from where they are grabbing my data really."[P7 in the contexts of voice agents and news feeds]

"Recently, I've been getting a lot of recommendation for music related to focus music, work music, some jazz music etc. I've never used (sic) those kinds of music before. So I feel like- Oh my God, where did this come from?" [P12 in the entertainment context]

Participants also mentioned that there is lack of knowledge and transparency in how their data is being used by these applications. Although they mentioned having agreed to terms and conditions of what data was collected or shared, they were more interested in knowing how that data is being used and would like to have more transparency on that front.

"I'm not saying what it tracked but I'm more interested in how it's being used. For example, it's tracking information of when, what and where I purchase something on Amazon, but then is it feeding into a third-party organization or how is that information being used?" [P13 in the context of e-commerce apps] "Sometimes even in incognito mode where you think it's not collecting or there are no cookies, but there are cookies and they know exactly what you're doing. I would really appreciate transparency in the sense of how apps collect and store my data. I do not want it storing all my information and you know throwing suggestions in my face." [P6 in maps and entertainment contexts]

\subsection{Shifting control}

Users had mixed views on having control. In the context of a smart thermostat (C4), P5 responded saying-

"I have the feeling I have a lot of control although it sometimes makes decisions for me. It's very easy also to take over control and that's one positive thing. So even if it makes decisions for you, it's very easy to take over control or overrule it. So that's why it was not that annoying for me."

On the other hand when it came to social media applications (C3), users perceived a lack of control over their feed and information they received.

"It feels like there is somebody above your head. It's a Puppet Master trying to control your actions and control your movements." [P15]

For some users it was not just about having control over the system but also about the degree of control they perceived to possess-

"I miss some degree of control, because I don't think I can switch off recommendations there. I can say stop recommending from this channel but not stop recommending these videos because I would probably still be interested in that channel, but I don't want YouTube to dig up five-year-old interview from that person and give it to me." [P12]

\subsection{Freedom of choice}

Users perceived a certain restriction in terms of freedom of choice. They felt that choices were there but it was harder to identify and search through all of them because the algorithms streamline them extensively.

"It's too much work to go and bypass these automation engines. The restriction is not in terms of options, but the restriction is in terms of making life difficult to figure out your own options. ECommerce sites take advantage of your wish list and your saved items. They kind of push you to buy those for example if you keep them for a few days. It restricts a more broader (sic) search in those cases because you are pushed a certain spectrum of products to look for. So in a sense it constrains the options that I could have looked for." [P14]

A similar opinion was also reflected in the entertainment context.

"Sometimes it is like I'm just looking at the same genre or same type of TV series or something like that. And sometimes I would like to see documentaries or other things. And it doesn't give me an option. Well, I mean, the choice is all there somewhere, but it doesn't show me. So it's kind of restricted." [P8]

"I would say these recommendations are quite biased on what I watched before but I am the kind of a person who likes to explore new content and it becomes difficult when I am being given the same sort of content that I've been watching over and over again. I feel that my thoughts also get biased in the same way." [P4] 


\subsection{Interconnected systems and data sharing}

Users also expressed concerns over applications using data from other applications or sharing its data with other platforms and applications. While most participants gave the example of targeted advertisements appearing across different application contexts, some of them also referred to applications directly using the data for other uses apart from advertising.

"I'm very scared that what I search on Google is something that Amazon or Makemytrip has access to and they are suggesting what I want to do. We are looking at a scary world where these people know what you want or where you want to travel and then make recommendations for that. I'm not sure what the solution is. I'm not sure where things are going but yeah, it looks quite scary." [P3]

"..all these things are kind of happening in the background, which I don't get to see and I am not being told up front. For example, I browsed something on Facebook and the next day I got something related to that particular thing on Instagram!” [P14]

\subsection{Trust and privacy}

A number of users expressed concerns regarding trust and privacy. While privacy concerns were related to cookies, tracking and the sheer amount of data being collected by these application, the lack of trust was well expressed in phrases such as "steal your data", "in a stealth way", or even more explicitly saying "I don't trust these companies".

"What is kind of unsettling is that they have been doing this for a few years now without the knowledge of the users. I don't think many people like me were consciously aware that companies are, kind of in a stealth way, gathering a lot of information on my personal interests and hobbies etc." [P15 in the social media context]

"Now you have GDPR and you have to click on agree in every website, but it's not like everyone has the patience to read through it or change the settings. I make sure as much as possible to disagree to everything, but sometimes I can't help but just clicking on 'agree all' because I am so much interested in reading the article. They make it difficult for you and there is still a lot of secretive ways to steal your data. So I am not very comfortable." [P4 in the context of news consumption (C9)]

\subsection{Manipulation or polarization}

In certain contexts such as a fitness application, users were more flexible with the application directing them towards their goals and actions since it is probably why they signed up for the application in the first place. However, in sensitive and personal contexts like social or political orientation, people preferred to be able to determine their own goals and actions and not be manipulated by the applications.
"I don't think I want to be labeled into boxes. My interests, my political interests, my hobbies- they don't define who I am and what I want to watch or do in future, so I want to kind of have the universe open." [P10 in the social media context]

“..it is a threat to our own independent way of thinking I think because there's a lot of content that is pushed to you and you rarely have the time to think for yourself." [P9 in news context]

"..people have extremely strong opinions and they keep reinforcing these stronger opinions again, and again. Especially these AI mediums which basically want to ensure that you keep clicking on the article and so they keep suggesting articles which they "know" will strongly evoke a reaction from you. And you keep reading more of that, you keep moving away from the center and that is something dangerous for society as a whole because people keep getting more polarized." [P3 in the news context]

\subsection{Breaking expectations}

Some users also stated that they encountered experiences where applications occasionally presented the user with something which was not expected by the user. In some contexts these behaviors left the users perplexed or annoyed.

“..after a few weeks. It [smart thermostat] started turning on at random moments. So I was like why is it turning on now, but you have no clue of course! There's something behind it taking over control. But the annoying part for me was it does it and I have to cancel it." [P5 in the context of domotics]

"What is frustrating is, it asked me if it was hard, easy or okay and I said it was hard. But it again kept giving me harder exercises. It was like it is not listening to me... takes the fun away from exercising." [P1 in the fitness context]

"I was so surprised because I have never done any donations for [a cause] through my mobile apps for anything but through my personal internet banking for the last two to three months, I have been donating for [the cause] separately. I was so surprised as how this ad is coming to me in the application. And sometimes I get so irritated." [P2]

\section{DISCUSSION}

Based on the insights from the phenomenological study, we reflect upon how the gathered user perceptions contribute to address our research questions- (RQ1) How do users perceive the overall experience of interacting with everyday AI applications, (RQ2) How did different contexts or application domains influence their experience, and (RQ3) Were they aware of the presence of an AI algorithm driving the apps and if they understood the functioning of these algorithms. We discuss these questions from the lens of personal autonomy. We also reflect upon implications of these 
findings on further research in human autonomy perspectives when designing AI applications.

\subsection{From the lens of personal autonomy}

As defined earlier, personal autonomy includes various components such as freedom of choice [33, 57], ability to determine one's own goals and actions $[14,48]$, having control and being able to make independent decisions $[1,4]$ and trust $[1,13,42]$. While we can draw some direct answers on these factors from the related meaning units (MU2, MU5, MU6, MU8), we can draw further meaningful inferences from other significant statements collected during the study. For example, time-saving (MU1) is not defined in theory as a component of personal autonomy. Nonetheless, in the context of interacting with technologies, users felt that as algorithms take over redundant and repetitive tasks and save time, they regain the ability (autonomy) to carry out other meaningful tasks.

4.1.1 RQ1: User experience of interacting with Al apps. Users in general had mixed opinions on their interaction experience with AI applications as was evident from the wide variety of sentiments that were conveyed through the extracted significant statements.

A. Experiences are ephemeral. A gradual shift in people's perspectives were reported alluding to the fact that these experiences are transitory and could change or evolve over time based on their understanding and socialization with technology.

"I would say my control over these applications, the data, is kind of slowly, gradually fading away. I'm having to do more effort each time I open an app to actually go off track from the feed shown to me." [P15]

"It does a good job at recommending the things that I like. So I think I'm okay with it, even though I wish that I could take it [referring to personal data] back, go back in time and stop it from doing it. But now I just know that there's no way I can do it." [P12]

"Now, I feel like I have lost control and I'm more getting into this sort of web." [P6]

While they had positive experiences of using and interacting with these applications, they are gradually coming to realize and understand certain negative aspects which leaves them feeling confused.

B. Re-asserting the power relation. Certain other experiences imply that people still want to be on top and not feel threatened by algorithms or systems. This was apparent when users said things like “..it's very easy to take over control or overrule it." One user also remarked on the lack of perfection in current AI applications which gave them a sense of relief and a feeling of winning over the machine:

"Maybe there is some amount of AI that needs to be fixed. For example, say if I'd ordered something for dinner, say pizza, sometimes it (referring to a food ordering app) recommends the same thing for breakfast as well, which is probably not the right match, but it's still giving that recommendation to me. It's funny right now. They still can't get their analytics right! And I'm happy they can't because I'll be scared if they start getting it too right!"
These observations resonate with the findings by Torkamann et al. [53] who examined the perception of creepiness in recommender systems which is associated with unwanted personalization, too accurate recommendations, and lack of control and transparency over data collection.

4.1.2 RQ2: Contextual influences on user perception. Prior research showed that in very specific contexts such as robotic shopping assistants or social robots, people disliked robots exhibiting a high degree of autonomy and often felt a loss of trust and control [45, 47]. In this paper, we tried to assess these perceptions from 'lived' everyday experiences of people to understand how their perceived autonomy varied across interactions in everyday contexts.

A. Privacy consciousness. In contexts where users did not have to divulge a lot of personal data such as entertainment, navigation, domotics, and booking applications, participants' responses indicated a higher degree of perceived autonomy. In most cases, they felt that the recommendations they received were based on their own preferences or choices (i.e subscribing to specific channels or genres on entertainment apps and setting personal preferences or filters in the applications). On the contrary, they perceived a relatively low degree of autonomy in contexts where more personal, political and privacy-sensitive data was involved such as social media or news feeds. Here, people often felt that the degree of control they have was gradually fading.

Furthermore, participants also expressed a lack of trust in these applications based on recent press and popular media reports highlighting privacy and ethical issues associated with these applications.
“..there's this constant sense of fear. Maybe it is be- cause I'm reading more about it (AI) and watching so many shows that it has gotten instilled that you know, it's obviously more than what you think it is."[P10]

In the past, studies have shown that both online and offline news and media reports have a powerful influence on people's opinions across various topics such as politics, drug use or environment [9, 50]. However, the specific influence on AI perception has not been studied in detail. Given the myriad of articles in the media about AI ranging from fear-mongering ones of robotic apocalypse or machines taking over jobs, to positive ones about the advances in disease predictions and prevention [38, 54]; it will be interesting to assess the influence that these articles have on user perceptions.

B. Optimising beyond human values and expectations. The concepts of echo chambers and filter bubbles have been extensively studied and researchers have demonstrated users' dilemma between privacy and personalization $[5,43]$. In our study we found that even in non-privacy-sensitive contexts such as entertainment, some users were riled by 'hyper' personalization and modeling and expressed views such as - "I don't want to be labelled in boxes" or ".. they don't define who I am as a person”. To address such concerns, adopting approaches such as "controllable" personalization as suggested by Bakalov et al. [3] could potentially be an effective strategy to enhance users' perception of autonomy. The potential of past behaviour to limit the serendipity and variety of future interactions with technology in non-media related contexts, has not received 
similar attention. The extent to which such funneling of user behaviour and interactions occurs and is experienced as a threat to autonomy, is an interesting question for future research.

Few scholars have studied the context-sensitive nature of AIbased recommendations upon perceived autonomy. In the field of marketing, similar context sensitivity has been reported [29], and was attributed to whether consumers derive identity relevant benefits from consuming a product, in which case they are more resistant to adopt algorithmic assistance that undermines the attribution of product choices to their own identity (e.g., in fashion). Future research could examine whether this is a manifestation of a more general phenomenon (beyond consumption) regarding the relation between the conception of one's self and the use of AI to support choice behaviour, or automation more generally.

Furthermore, participants experienced a perception of reduced autonomy and felt annoyed when certain expectations were broken. This is especially the case when their interactions with applications are contrasted with human-human interactions. An example discussed above, is the case of a fitness app which does not recommend easier workouts when the user complains they are too hard. From a technical standpoint, we could argue that the application will need more data points before it can make the expected adaptations. However, this is not apparent to naive users, leaving them frustrated. Haberland et al. [19] call this lack of naturalness in the interaction with machines as an asymmetric interaction which often makes computers unpredictable and has given them a kind of power that users cannot change. Therefore, from the lens of autonomy, this asymmetry in interactions forms yet another key consideration.

4.1.3 RQ3: Awareness and understanding of AI. The need for transparency and explainability in AI have been extensively studied. Early research focused primarily on algorithmic transparency and making machine learning models more interpretable [6, 37]. From a human-centered perspective, researchers have also looked into aspects such as cognitive biases and social expectations to identify what constitutes a good explanation to inform design practices to improve user experience [30,36]. Yet, Ngo et al. [41], argued that not much has been done to investigate users' perceptions and understanding of these applications or explanations. They also showed that in an everyday context such as when using Netflix, participants exhibited a high level of uncertainty and confusion about the recommender system. Our findings extend this observation to users' understanding of applications in different everyday contexts and suggests that they also needed a certain degree of control over their data apart from simple explanations.

A. Need for intelligibility. Participants were aware of some types of data which was being used such as their preferences, cookies and browsing, search or activity history. Yet, they lacked an understanding of how their personal data was used.

"I can no longer distinguish between what is being pushed to me and what is based on my history." [P4]

This also indicates a need for intelligibility, i.e., for users to be able to comprehend 'why' or 'why not' certain recommendations were made [32].

B. Control over data. Participants also felt that they often times unconsciously divulge information they did not intend to because of the lack of transparency and lack of a user-friendly approach to control or secure their data.

"I just want to be a passive user. It's a busy life and I think I don't give it too much of a thought when I'm actually doing this [browsing through social media feed]. But suddenly I get uncomfortable thinking that what I'm doing is not a passive exercise. It's very active. They are collecting my data and there's nothing that's passive." [P10]

In order to gain more control, participants indicated that they try to over-rule the algorithms by adopting different strategies such as "I don't click on the recommendations", "I go incognito", and "I un-link my apps and devices". Three of the more tech-savvy participants also mentioned that they do not even trust incognito modes and use alternative browsers and search engines. P14 also remarked that they will be willing to pay a premium subscription to be able to get more control over these applications.

Despite these there was a sense of skepticism and lack of trust amongst all participants which is reflected well in this statement of P3-

“..it's still a black box and no matter what they do, no matter how transparent they make it, unless they clearly specify what they want to do and unless they make it very easy for you to say- No; as easy as it is today to say- Yes; instead of wrapping it around a very complex structure, I will not get any comfort from them."

In essence, the experiences shared by participants indicate that while some users still perceive a reasonable sense of personal autonomy when interacting with these applications, they feel that their autonomy is gradually ebbing away and seem worried about it. Especially, experiences where they feel manipulated, or when they retrospectively realize that they only had an illusion of control and were potentially coerced into decisions without being presented with all options, they feel like a puppet with an invisible and unintelligible AI tugging at the strings. It is therefore important as researchers and designers of human-centered AI to be aware of these perspectives to design and model applications that respect users' perceptions of personal autonomy.

\subsection{Directions for future research}

The need to focus on human autonomy and to study user perceptions and understanding of AI in everyday contexts have been deemed critical for the future development of human-centered AI $[25,41]$. Our study forms a step in this direction where we identified key facets of human autonomy as experienced by users in everyday interactions with AI. All participants in our study had at least a bachelor's degree. Future research could examine how people with less education experience loss of autonomy to AI. While some of the autonomy facets identified in our study have been explored in prior research, a few critical aspects remain to be explored more extensively [Figure 2]. Based on this inference, we highlight three key directions for future research.

4.2.1 Symbiotic autonomy. Increasingly, a greater emphasis is being laid on the need to explore the interplay between humans and 


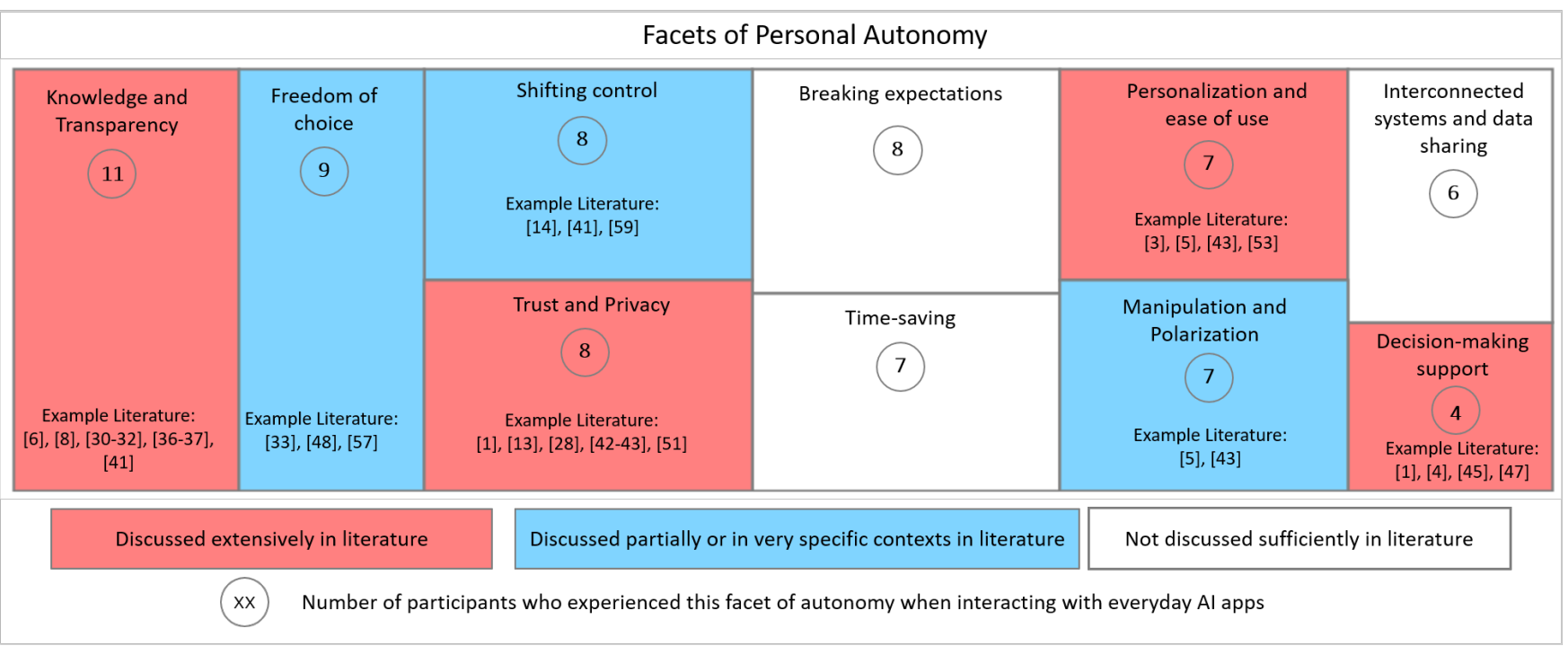

Figure 2: Facets of human autonomy as based on the meaning units identified in this study highlighting areas that are underexplored in literature.

$\mathrm{AI}$ in order to build systems that do not undermine human values and performance [24]. To this end researchers have been working towards what is called hybrid intelligence [27]. At the foundation of identifying complimentary and bi-directional interaction between humans and AI, finding a balance on autonomy is the key. Veloso et al. [56] have been conducting experiments with service robots where robots proactively seek the help of humans to carry out certain tasks and exhibit what they term as symbiotic autonomy. Similar approaches could be extended into everyday application contexts to identify how we could design symmetric interactions between humans and AI without inhibiting the users' conception of self and perception of autonomy.

4.2.2 Intelligibility over explainability. Data scientists are studying the trade-offs and effectiveness of transparent glass-box models versus explainable black-box models in a drive towards making machine learning more intelligible and interpretable [8]. From a user-perspective, research has shown that intelligibility can help users understand complex, context-aware systems [31]. We identified that knowledge and understanding of applications as well as perceptions of privacy and trust form a key factor in perceptions of autonomy. Abdul et al. [2] carried out an extensive literature analysis to identify current trends and research opportunities for explainable systems. Specifically, the following three trajectories they recommend could facilitate in enhancing human autonomy through intelligibility- (i) exploring interactive explanations and interfaces for intelligibility, (ii) working towards improving usability and rigor of the explanations, and (iii) designing explanations to go beyond gaining individual trust to focus on more systematic accountability.

4.2.3 Evolving experiences. Users' experiences and expectations vary in different contexts, and are constantly evolving based on various internal and external influences. Similarly, autonomy is not a singular metric but rather it encompasses various factors. There are also various degrees of autonomy and it is therefore critical to identify the level of autonomy users seek in a specific contexts. Schneiderman [51] highlighted how trust and reliability could vary depending on degrees of human control and machine autonomy. In his pioneering work, Pearl has argued that causal reasoning is an indispensable part of human thought and provides a structured computational framework and set of tools for causal inference [44]. Specifically, the method for causal discovery [52], which is based on the detection of 'shocks' or changes in the interaction environment, could be key in identifying how the external factors are influencing people's perceptions and experiences. Therefore, future research could focus more on exploring cause-effect connections and how that influences the degree of perceived autonomy. Furthermore, given the temporal nature of human experiences, it is crucial to iteratively re-evaluate these experiences as human-AI interactions evolve.

\section{CONCLUSION}

In this paper, we explored user perceptions of personal autonomy when interacting with everyday AI applications. We presented a broad view on various facets of personal autonomy and delved into everyday use contexts and experiences which are under-explored in literature. We adopted a phenomenological approach to capture the essence of user experiences across different application contexts. The outcomes suggest that user experiences and perceptions are transitory and influenced by external factors such as media reports. People perceive a lower degree of autonomy in privacy-sensitive contexts or when AI algorithms cross a context and break user expectations or counter conceptions of one's own identity. The study showed that users would appreciate more intelligibility in understanding how their data is being used and would like to have the ability to control or over-rule the decisions or actions of algorithms 
and thereby not feel like a puppet being manipulated by AI. The desirable levels of control and autonomy are context dependent. Future research may delve deeper into whether the willingness of people to accept AI may depend on how their conception of their own identity and autonomy is threatened or not and by emphasizing a symbiotic relationship between AI and its human users, as a key dimension of the user experience. By emphasizing the need and importance of respecting human autonomy in human-AI interactions, we believe that the insights and user perceptions presented in this paper will open up further discussions and will trigger further research into designing and developing AI applications which strive to safeguard users' sense and experience of autonomy.

\section{REFERENCES}

[1] Hussein A Abbass. 2019. Social integration of artificial intelligence: functions, automation allocation logic and human-autonomy trust. Cognitive Computation 11, 2 (2019), 159-171.

[2] Ashraf Abdul, Jo Vermeulen, Danding Wang, Brian Y Lim, and Mohan Kankanhalli. 2018. Trends and trajectories for explainable, accountable and intelligible systems: An hci research agenda. In Proceedings of the 2018 CHI conference on human factors in computing systems. 1-18.

[3] Fedor Bakalov, Marie-Jean Meurs, Birgitta König-Ries, Bahar Sateli, René Witte, Greg Butler, and Adrian Tsang. 2013. An approach to controlling user models and personalization effects in recommender systems. In Proceedings of the 2013 international conference on Intelligent user interfaces. 49-56.

[4] K Suzanne Barber and Cheryl E Martin. 2000. Autonomy as decision-making control. In International Workshop on Agent Theories, Architectures, and Languages. Springer, 343-345.

[5] Pinar Barlas, Kyriakos Kyriakou, Antrea Chrysanthou, Styliani Kleanthous, and Jahna Otterbacher. 2020. OPIAS: Over-Personalization in Information Access Systems. In Adjunct Publication of the 28th ACM Conference on User Modeling, Adaptation and Personalization. 103-104.

[6] Vaishak Belle and Ioannis Papantonis. 2020. Principles and practice of explainable machine learning. arXiv preprint arXiv:2009.11698 (2020).

[7] Christopher Burr, Nello Cristianini, and James Ladyman. 2018. An analysis of the interaction between intelligent software agents and human users. Minds and machines 28, 4 (2018), 735-774.

[8] Rich Caruana, Scott Lundberg, Marco Tulio Ribeiro, Harsha Nori, and Samuel Jenkins. 2020. Intelligible and Explainable Machine Learning: Best Practices and Practical Challenges. In Proceedings of the 26th ACM SIGKDD International Conference on Knowledge Discovery \& Data Mining. 3511-3512.

[9] Cindy T Christen and Kelli E Huberty. 2007. Media reach, media influence? The effects of local, national, and Internet news on public opinion inferences. fournalism \& Mass Communication Quarterly 84, 2 (2007), 315-334.

[10] Paul F. Colaizzi. 1978. Psychological Research as the Phenomenologist Views It. In Existential-Phenomenological Alternatives for Psychology, Ronald S. Valle and Mark King (Eds.). Oxford University Press, 6.

[11] European Commission. 2020. White Paper on Artificial Intelligence: a European approach to excellence and trust. https://ec.europa.eu/info/publications/whitepaper-artificial-intelligence-european-approach-excellence-and-trust_en

[12] John W Creswell and Cheryl N Poth. 2016. Qualitative inquiry and research design: Choosing among five approaches. Sage publications.

[13] Ewart J de Visser, Richard Pak, and Tyler H Shaw. 2018. From 'automation'to 'autonomy': the importance of trust repair in human-machine interaction. $E r-$ gonomics 61, 10 (2018), 1409-1427.

[14] Edward L Deci and Richard M Ryan. 1987. The support of autonomy and the control of behavior. Fournal of personality and social psychology 53, 6 (1987), 1024.

[15] Sheree Dukes. 1984. Phenomenological methodology in the human sciences. Journal of religion and health 23, 3 (1984), 197-203.

[16] Joseph L Fleiss, Bruce Levin, Myunghee Cho Paik, et al. 1981. The measurement of interrater agreement. Statistical methods for rates and proportions 2, 212-236 (1981), 22-23.

[17] Richard M Frankel. 1999. Standards of qualitative research (2 ed.). sage publications.

[18] Amedeo Giorgi. 1985. Phenomenology and Psychological Research. Duquesne University Press. Google-Books-ID: JpkQAQAAIAAJ.

[19] Hartmut Haberland. 1999. The natural and the artificial in language and technology. In Humane interfaces: questions of method and practice in cognitive technology. Pergamon Press, 81-87.

[20] Marc Hassenzahl and Noam Tractinsky. 2006. User experience-a research agenda. Behaviour \& information technology 25, 2 (2006), 91-97.
[21] Edmund Husserl. 2012. Ideas: General introduction to pure phenomenology. Routledge.

[22] Don Ihde. 1995. Postphenomenology: Essays in the postmodern context. Northwestern University Press.

[23] Don Ihde. 2012. Experimental phenomenology: multistabilities. Suny Press.

[24] Kori Inkpen. 2020. Does My AI Help or Hurt? Exploring Human-AI Complementarity. In Proceedings of the 28th ACM Conference on User Modeling, Adaptation and Personalization. 2-2.

[25] Lee Rainie Janna Anderson and Alex Luchsinger. 2018. Artificial Intelligence and the Future of Humans. https://www.pewresearch.org/internet/2018/12/10/ artificial-intelligence-and-the-future-of-humans/

[26] Adrian L. Van Kaam. 1966. Existential Foundations of Psychology. Duquesne University Press. Google-Books-ID: OA2dAAAAMAAJ.

[27] Ece Kamar. 2016. Directions in Hybrid Intelligence: Complementing AI Systems with Human Intelligence.. In I7CAI. 4070-4073.

[28] Matthias Kraus, Nicolas Wagner, and Wolfgang Minker. 2020. Effects of Proactive Dialogue Strategies on Human-Computer Trust. In Proceedings of the 28th ACM Conference on User Modeling, Adaptation and Personalization. 107-116.

[29] Eugina Leung, Gabriele Paolacci, and Stefano Puntoni. 2018. Man versus machine: Resisting automation in identity-based consumer behavior. fournal of Marketing Research 55, 6 (2018), 818-831.

[30] O Vera Liao, Daniel Gruen, and Sarah Miller. 2020. Questioning the AI: Informing Design Practices for Explainable AI User Experiences. In Proceedings of the 2020 CHI Conference on Human Factors in Computing Systems. 1-15.

[31] Brian Y Lim and Anind K Dey. 2013. Evaluating intelligibility usage and usefulness in a context-aware application. In International Conference on Human-Computer Interaction. Springer, 92-101.

[32] Brian Y Lim, Anind K Dey, and Daniel Avrahami. 2009. Why and why not explanations improve the intelligibility of context-aware intelligent systems. In Proceedings of the SIGCHI Conference on Human Factors in Computing Systems. 2119-2128.

[33] Michael Luck, Mark d'Inverno, et al. 1995. A Formal Framework for Agency and Autonomy. In ICMAS, Vol. 95. AAAI Press, 254-260.

[34] Lynn M Meadows and Janice M Morse. 2001. Constructing evidence within the qualitative project. The nature of qualitative evidence (2001), 187-200.

[35] Matthew B. Miles, A. Michael Huberman, and Johnny Saldana. 2014. Qualitative Data Analysis. SAGE. Google-Books-ID: 3CNrUbTu6CsC

[36] Tim Miller. 2017. Explanation in artificial intelligence: Insights from the social sciences. CoRR abs/1706.07269 (2017). arXiv preprint arXiv:1706.07269 (2017)

[37] Christoph Molnar. 2020. Interpretable Machine Learning. Lulu. com.

[38] Alex Moltzau. 2019. AI - The Good, The Bad and The Ugly. https://medium. com/odscjournal/ai-the-good-the-bad-and-the-ugly-ec7d08f9fa11

[39] Clark Moustakas. 1994. Phenomenological research methods. SAGE Publications, Inc., 2455 Teller Road, Thousand Oaks California 91320 United States of America. https://doi.org/10.4135/9781412995658

[40] Brian E Neubauer, Catherine T Witkop, and Lara Varpio. 2019. How phenomenology can help us learn from the experiences of others. Perspectives on medical education 8, 2 (2019), 90-97.

[41] Thao Ngo, Johannes Kunkel, and Jürgen Ziegler. 2020. Exploring Mental Models for Transparent and Controllable Recommender Systems: A Qualitative Study. In Proceedings of the 28th ACM Conference on User Modeling, Adaptation and Personalization. 183-191.

[42] High-Level Expert Group on Artificial Intelligence. 2019. Ethics guidelines for trustworthy AI. European Commission (2019).

[43] Saurabh Panjwani, Nisheeth Shrivastava, Saurabh Shukla, and Sharad Jaiswal. 2013. Understanding the privacy-personalization dilemma for web search: a user perspective. In Proceedings of the SIGCHI Conference on Human Factors in Computing Systems. 3427-3430.

[44] Judea Pearl. 2019. The seven tools of causal inference, with reflections on machine learning. Commun. ACM 62, 3 (2019), 54-60.

[45] Zhenhui Peng, Yunhwan Kwon, Jiaan Lu, Ziming Wu, and Xiaojuan Ma. 2019. Design and evaluation of service robot's proactivity in decision-making support process. In Proceedings of the 2019 CHI Conference on Human Factors in Computing Systems. 1-13.

[46] Donald E Polkinghorne. 1989. Phenomenological research methods. In Existentialphenomenological perspectives in psychology. Springer, 41-60.

[47] Pei-Luen Patrick Rau, Ye Li, and Jun Liu. 2013. Effects of a social robot's autonomy and group orientation on human decision-making. Advances in Human-Computer Interaction 2013 (2013).

[48] Richard M Ryan and Edward L Deci. 2006. Self-regulation and the problem of human autonomy: Does psychology need choice, self-determination, and will? Journal of personality 74, 6 (2006), 1557-1586.

[49] Supraja Sankaran, Chao Zhang, Mathias Funk, Henk Aarts, and Panos Markopoulos. 2020. Do I Have a Say? Using Conversational Agents to Re-Imagine HumanMachine Autonomy. In Proceedings of the 2nd Conference on Conversational User Interfaces (Bilbao, Spain) (CUI '20). Association for Computing Machinery, New York, NY, USA, Article 18, 3 pages. https://doi.org/10.1145/3405755.3406135 
[50] Frank W Schneider, Jamie A Gruman, and Larry M Coutts. 2011. Applied social psychology: Understanding and addressing social and practical problems. Sage.

[51] Ben Shneiderman. 2020. Human-centered artificial intelligence: Reliable, safe \& trustworthy. International fournal of Human-Computer Interaction 36, 6 (2020), 495-504.

[52] Jin Tian and Judea Pearl. 2002. A general identification condition for causal effects. In Aaai/iaai. 567-573.

[53] Helma Torkamaan, Catalin-Mihai Barbu, and Jürgen Ziegler. 2019. How can they know that? A study of factors affecting the creepiness of recommendations. In Proceedings of the 13th ACM Conference on Recommender Systems. 423-427.

[54] Lauren Tousignant. 2017. 5 terrifying stories that warn of an AI apocalypse. https://nypost.com/2017/12/28/terrifying-stories-that-prove-the-aiapocalypse-is-imminent/
[55] Max Van Manen. 2016. Researching lived experience: Human science for an action sensitive pedagogy. Routledge.

[56] Manuela M Veloso. 2018. The increasingly fascinating opportunity for humanrobot-ai interaction: The cobot mobile service robots.

[57] Anastasia Vugts, Mariëtte Van Den Hoven, Emely De Vet, and Marcel Verweij. 2018. How autonomy is understood in discussions on the ethics of nudging. Behavioural Public Policy (2018), 1-16.

[58] Lana Wachowski and Lilly Wachowski. 2003. The Matrix Reloaded.

[59] Gina Wessel, Eugen Altendorf, Constanze Schreck, Yigiterkut Canpolat, and Frank Flemisch. 2019. Cooperation and the role of autonomy in automated driving. In Control Strategies for Advanced Driver Assistance Systems and Autonomous Driving Functions. Springer, 1-27.

[60] Fareed Zakaria. 1997. The rise of illiberal democracy. Foreign Aff. 76 (1997), 22. 